\title{
CONSTRUCTING ORDERS BY BLOWING-UP
}

\author{
By \\ Yuji Yoshino and Yukihiro OsA
}

In his paper [1], M. Artin succeeded to classify maximal orders of global dimension and Krull dimension two having the formal power series rings over $\boldsymbol{C}$ as centers. In his method of classification, [1, Theorem (2.15)] is basic, in which he constructs a new order from a given maximal order and an extension of the center. In the present paper we reconstruct his orders avoiding several assumptions in his theorem. It will be done by defining the blowing-up, see Definition below. We are inspired this by [2]. The point is that we can define it globally, and that we can clarify what was really necessary in Artin's theorem.

Throughout this paper $R$ is a Noetherian normal domain and $K$ is the field of quotients of $R$. Let $L$ be a finite field extension of $K$ and let $S$ be the integral closure of $R$ in $L$. We always assume that $S$ is module-finite over $R$. Furthermore $\Sigma$ is a $K$-Azumaya algebra and $A$ is an $R$-order in $\Sigma$.

We denote by $X^{1}(R)$ (resp. $X^{1}(S)$ ) the set of prime ideals in $R$ (resp. $S$ ) of height one. For any element $\mathfrak{B} \in X^{1}(S)$ with $\mathfrak{p}=\mathfrak{P} \cap R$, we define as follows:

$$
\begin{aligned}
& J_{\mathfrak{p}}=\operatorname{rad} A_{\mathfrak{p}} \cap A, \\
& r_{\mathfrak{B}}=\min \left\{n \mid \mathfrak{P}^{n} S_{\mathfrak{P}} \subset \mathfrak{p} S_{\mathfrak{p}}\right\}=\text { the ramification index of the extension } R_{\mathfrak{p}} \subset S_{\mathfrak{B}}, \\
& e_{\mathfrak{p}}=\min \left\{n \mid\left(\operatorname{rad} A_{\mathfrak{p}}\right)^{n} \subset \mathfrak{p} A_{\mathfrak{p}}\right\}=\text { the ramification index of } A_{\mathfrak{p}} .
\end{aligned}
$$

In what follows we assume that $A$ is a tame R-order, i.e. $A$ is a reflexive $R$-module and $A_{\mathfrak{p}}$ is a hereditary $R_{\mathfrak{p}}$-order for any $\mathfrak{p} \in X^{1}(R)$. Notice that maximal orders are tame. Since the set of $\mathfrak{p} \in X^{1}(R)$ with $e_{p} \neq 1$ is finite, denote it by $\left\{\mathfrak{p}_{1}, \mathfrak{p}_{2}, \cdots, \mathfrak{p}_{l}\right\}$. For each $i(1 \leqq i \leqq l)$, we denote by $\left\{\mathfrak{P}_{i 1}, \mathfrak{P}_{i 2}, \cdots, \mathfrak{P}_{i_{i}}\right\}$ the set of prime ideals of $S$ which lie over $\mathfrak{p}_{i}$. For simplicity we write $e_{i}=e_{\mathfrak{p}_{i}}, r_{i j}=$ $r_{\Re_{i j}}$ and $J_{i}=J_{\mathfrak{p}_{i}}$ for $1 \leqq i \leqq l, 1 \leqq j \leqq \nu_{i}$. Furthermore we denote $s_{i j}=e_{i} / r_{i j} \in \boldsymbol{Q}$.

Definition. We define the blowing-up $B(A, S)$ of $A$ along $S$ by the following :

$$
B(A, S)=\sum_{\left(n_{1}, n_{2}, \cdots, n_{l) \in Z^{l}}\right.}\left\{\prod_{i=1}^{l}\left(J_{i}^{-n_{i}} \prod_{j=1}^{\nu_{i}} \Re_{\left.i j^{[n} i_{i} / s_{i j}\right]}\right)\right\}^{* *} \subset \Sigma \otimes_{K} L,
$$

where ()$^{* *}=\operatorname{Hom}_{R}\left(\operatorname{Hom}_{R}(, R), R\right)$ and $[q]=\min \{n \in \boldsymbol{Z} \mid n \geqq q\}$ for any $q \in \boldsymbol{Q}$. Received July 10, 1990. 
REMARK. Since $\left[n_{i} / s_{i j}+n_{i}^{\prime} / s_{i j}\right] \leqq\left[n_{i} / s_{i j}\right]+\left[n_{i}^{\prime} / s_{i j}\right]$, it is easily seen that $B(A, S)$ is closed under multiplication. Hence $B(A, S)$ is an $S$-algebra and $B(A, S) \otimes_{S} L=\Sigma \otimes_{K} L$. We also define that, if $e_{\mathfrak{p}}=1$ for any $\mathfrak{p} \in X^{1}(R)$ (i. e. $l=0$ ), then $B(A, S)=A \otimes_{R} S$.

LEMMA 1. $B(A, S)$ is finitely generated as an S-module.

Proof. Remarking that $\left[\left(n_{i}+e_{i}\right) / s_{i j}\right]=\left[n_{i} / s_{i j}\right]+r_{i j}$, we have

$$
\mathfrak{P}^{\left[\left(n_{i}+e_{i}\right) / s_{i j}\right]}=\mathfrak{P}^{\left[n_{i} / s_{i j}\right]} \cdot \mathfrak{P}^{r_{i j}} \text {. }
$$

Letting $B\left(n_{1}, n_{2}, \cdots, n_{l}\right)=\prod_{i=1}^{l}\left(J_{i}^{-n_{i}} \prod_{j}^{\nu} \underline{\underline{i}}_{1} \mathfrak{P}_{i j}^{\left[n_{i} / s_{i j}\right]}\right)$, we see from this that

$$
B\left(n_{1}, \cdots, n_{i}+e_{i}, \cdots, n_{l}\right)=B\left(n_{1}, n_{2}, \cdots n_{l}\right) \cdot\left(J_{i}^{-e_{i}} \prod_{j=1}^{\nu_{i}} \mathfrak{P}_{i j}^{r_{i j}}\right) .
$$

Here, since $\left.\left(J_{i}^{-e_{i}} \prod_{j=1}^{\mu_{i}} \Re_{i j}^{r}\right)^{r_{i j}}\right)^{* *}=A \bigotimes_{R} S$, we obtain

$$
B(A, S)=\sum_{\left(n_{1}, n_{2}, \cdots, n_{l}\right) \in \boldsymbol{Z}^{l}, 0 \leq n_{i}<e_{i}} B\left(n_{1}, n_{2}, \cdots n_{l}\right)^{* *} .
$$

Each $B\left(n_{1}, n_{2}, \cdots, n_{l}\right)^{* *}$ is a finitely generated $S$-module, therefore $B(A, S)$ is.

If we denote the center of $B(A, S)$ by $Z$, then clearly $S \subset Z \subset L$ and $Z$ is finitely generated as an $S$-module by the lemma. Hence we have $S=Z$, showing the following:

Proposition 1. $B(A, S)$ is an S-order in $\Sigma \otimes_{K} L$.

We note that blowing-ups are preserved by certain base changes. More precisely we can see the following lemma whose proof is left to the reader.

LEMMA 2. (2.1) If $U$ is a multiplicatively closed subset of $S$ and if $V=U$ $\cap R$, then $B(A, S)_{U}=B\left(A_{V}, S_{U}\right)$.

(2.2) If $R \rightarrow R^{\prime}$ is a finite étale extension, then $B(A, S) \otimes_{R} R^{\prime}=B\left(A \otimes_{R} R^{\prime}\right.$, $\left.S \otimes_{R} R^{\prime}\right)$.

(2.3) If both $R$ and $S$ are discrete valuation rings, then $B(\hat{A}, \hat{S})=B(A, S) \otimes_{S} \hat{S}$.

In the rest of the paper we write $s_{i j}$ as

$$
s_{i j}=q_{i j} / p_{i j}, \quad q_{i j}, p_{i j} \in N \text { with }\left(p_{i j}, q_{i j}\right)=1 .
$$

Notice that, if $r_{i j}$ divides $e_{i}$, then $q_{i j}=s_{i j}=e_{i} / r_{i j}$, and that $q_{i j}=1$ if and only if $e_{i}$ divides $r_{i j}$. In general, $q_{i j}=e_{i} /\left(e_{i}, r_{i j}\right)$.

We can now state our main theorem of this paper.

THEOREM 1. Assume that $\mathfrak{\kappa}(\mathfrak{p})=R_{\mathfrak{p}} / \mathfrak{p} R_{\mathfrak{p}}$ is a perfect field for any $\mathfrak{p} \in X^{1}(R)$. 
Then $B(A, S)$ is also a tame $S$-order and, for any $\mathfrak{B} \in X^{1}(S)$, the ramification index of $B(A, S)$ at $\mathfrak{P}$ is equal to $q_{i j}$ if $\mathfrak{P}=\mathfrak{P}_{i j}$, and 1 otherwise.

Proof. Take $\mathfrak{B} \in X^{1}(S)$ and set $\mathfrak{p}=\mathfrak{B} \cap R$.

Suppose, first, that $\mathfrak{P}$ is distinct from any of $\mathfrak{P}_{i j}$. Then we see from Lemma 2 that $B(A, S)_{\mathfrak{P}}=B\left(A_{\mathfrak{p}}, S_{\mathfrak{P}}\right)=A_{\mathfrak{p}} \otimes_{\mathbb{R}_{\mathfrak{p}}} S_{\mathfrak{P}}$. Since $e_{\mathfrak{p}}=1$ and since $\kappa(\mathfrak{p})$ is perfect, we can see that $B(A, S)_{\mathfrak{P}}$ is an Azumaya algebra over $S_{\mathfrak{B}}$, in particular, it is hereditary with ramification index one.

Next assume that $\mathfrak{P}=\mathfrak{B}_{i j}$ for some $i, j$. For simplicity, set $r=r_{i j}, e=e_{i}$ and $s=s_{i j}$. By Lemma 2, we may assume that $(R, \mathfrak{p})$ and $(S, \mathfrak{B})$ are complete discrete valuation rings. Since $\kappa(\mathfrak{p})$ is perfect it is known by [4, Chap. XII $\S 1$ Theorem 1] that there is a finite étale extension $R \rightarrow R^{\prime}$ such that the quotient field of $R^{\prime}$ splits $\Sigma$, i.e. $\Sigma \otimes_{R} R^{\prime}$ is a matrix algebra over the quotient field of $R^{\prime}$. Considering $R^{\prime}$ instead of $R$ and using Lemma 2, we may assume that $\Sigma$ is a matrix algebra over $K$. Then by [3, Theorem (39.14)] we can find integers $m, m_{0}, m_{1}, \cdots, m_{e-1}$ with $m=\sum_{i=0}^{e-1} m_{i}$ and $R$-free modules of rank $m$;

$$
V_{i}=\mathfrak{p} \oplus \cdots \oplus \mathfrak{p} \oplus R \oplus \cdots \oplus R
$$

(there are $m_{0}+m_{1}+\cdots+m_{i-1} \mathfrak{p}$ 's in $V_{i}$ and $1 \leqq i \leqq e$ )

and the hereditary order $A$ is obtained as follows:

For any integer $n$, if $n=c e+d$ with $c, d \in Z$ and $1 \leqq d \leqq e$, then we define $V_{n}=\mathfrak{p}^{c} \bigotimes_{R} V_{d}$. Note that $\cdots \subset V_{n+1} \subset V_{n} \subset V_{n-1} \subset \cdots$. We denote this "flag" by $\boldsymbol{f}$ and, for any integer $n$, we write as follows:

$$
\operatorname{End}_{R}(\boldsymbol{f})_{n}=\left\{\varphi \in \operatorname{End}_{K}\left(V_{0} \bigotimes_{R} K\right) \mid \varphi\left(V_{i}\right) \subset V_{i+n} \text { for all } i\right\} .
$$

Then the hereditary $\operatorname{order} A$ is isomorphic to $\operatorname{End}_{R}(\boldsymbol{f})_{0}$ as an $R$-order. More generally, if $J$ is the Jacobson radical of $A$, then $J^{n}=\operatorname{End}_{R}(\boldsymbol{f})_{n}$ under this isomorphism.

For the proof of the theorem we need several auxiliary results.

Definition. For a rational number $a \in \boldsymbol{Q}$, we define the scaled Rees ring with center $\mathfrak{p}$ by

$$
R[\mathfrak{p}, a]=\sum_{n \in Z} \mathfrak{p}^{[n / a]} t^{n} \subset K\left[t, t^{-1}\right]
$$

We should notice the following:

LEMMA 3. Let $a=q / p$ be a rational number with $p, q \in \boldsymbol{N}$ coprime. If an integer $n$ has an equation $n=c q+d,(c, d \in \boldsymbol{Z}, 0 \leqq d<q)$, then $t^{n} R[\mathfrak{p}, a] \cong t^{d} R[\mathfrak{p}, a]$ as a graded $R[\mathfrak{p}, a]$-module. 
Furthermore, graded $R[\mathfrak{p}, a]$-modules $\left\{R[\mathfrak{p}, a], t^{1} R[\mathfrak{p}, a], t^{2} R[\mathfrak{p}, a], \cdots\right.$, $\left.t^{q-1} R[\mathfrak{p}, a]\right\}$ are non-isomorphic.

Proof. Since $t^{a} R[\mathfrak{p}, a]=\mathfrak{p}^{-p} R[\mathfrak{p}, a] \cong R[\mathfrak{p}, a]$ by definition, the first claim follows.

For the second, we define the function $\rho_{a}: Z \rightarrow N$ by the following:

Note that

$$
\boldsymbol{\rho}_{a}(i)=\#\{j \in \boldsymbol{Z} \mid[j / a]=i\} .
$$

$$
R[\mathfrak{p}, a]=\cdots \oplus R \oplus \cdots \oplus R \oplus \mathfrak{p} \oplus \cdots \oplus \mathfrak{p} \oplus \mathfrak{p}^{2} \oplus \cdots \oplus \mathfrak{p}^{2} \oplus \cdots,
$$

where each $\mathfrak{p}^{i}$ appears $\rho_{a}(i)$-times. It is, then, easy to see that Lemma 3 follows from the next lemma.

LEMMA 4. With the same notation as above, $p$ is the minimal period of the function $\rho_{a}$ and

$$
\sum_{i=0}^{p-1} \rho_{a}(i)=\#\{j \in \boldsymbol{Z} \mid 0 \leqq[p j / q]<p\}=q
$$

Proof of Lemma 4. It is obvious that $\rho_{a}(i+p)=\rho_{a}(i)$ for any $i \in \boldsymbol{Z}$. Let $s$ be the minimal positive integer with this property. Then clearly $s$ divides $p$. We have to show $s=p$. Letting $a^{\prime}=(p+q) / p$, we can see that $\rho_{a^{\prime}}(i)=$ $\rho_{a}(i)+1$ for any $i \in \boldsymbol{Z}$. Hence we may assume that $0<q<p$. Then $\rho_{a}$ takes either 1 or 0 , hence

$$
\begin{aligned}
\sum_{i=0}^{p-1} \rho_{a}(i) & =\#\left\{j \in Z \mid 0 \leqq j<p, \rho_{a}(j)=1\right\} \\
& =\#\{j \mid 0 \leqq j<q\}=q .
\end{aligned}
$$

On the other hand the leftmost of this equation is equal to $(p / s) \sum_{i=0}^{s-1} \rho_{a}(i)$, since $s$ is the period of $\rho_{a}$. Therefore $p / s$ divides $q$ and thus $p=s$.

Now we continue the proof of the theorem. For simplicity we write $\left(t^{i}\right)=$ $t^{i} R[\mathfrak{p}, e]$ and consider the $R[\mathfrak{p}, e]$-free module :

$$
\boldsymbol{V}=(1) \oplus(1) \oplus \cdots \oplus(1) \oplus(t) \oplus \cdots \oplus(t) \oplus\left(t^{2}\right) \oplus \cdots \oplus\left(t^{2}\right) \oplus \cdots \oplus\left(t^{e-1}\right),
$$

where each $\left(t^{i}\right)$ appears $m_{i}$-times. By the choice of $\left\{m_{i}\right\}$, there is an equality of graded rings:

$$
\operatorname{End}_{R[p, e]}(\boldsymbol{V})=\sum_{n \in Z} J^{n} t^{n} \subset \Sigma\left[t, t^{-1}\right] .
$$

Note that the hereditary $\operatorname{order} A$ is the part of degree 0 in this endomorphism ring, i. e. $A=\operatorname{End}_{R[\mathrm{p}, e]}(\boldsymbol{V})_{0}$.

Note also that there is an extension of commutative graded rings; $R[\mathfrak{p}, e]$ 
$\subset S[\mathfrak{P}, s]$, since $\mathfrak{p}^{[n / e]} \subset\left(\mathfrak{P}^{r}\right)^{[n / e]} \subset \mathfrak{P}^{[n / s]}$. Then by definition there is an equality :

$$
B(A, S)=\left(\sum_{n \in Z} J^{n} t^{n} \bigotimes_{R[\mathfrak{p}, e]} S[\mathfrak{P}, s]\right)_{0}
$$

Since $\boldsymbol{V}$ is a free $R[p, e]$-module, we obtain from this that

$$
B(A, S)=\left(\operatorname{End}_{S[\mathfrak{R}, s]}\left(\boldsymbol{V} \otimes_{R[p, e]} S[\mathfrak{P}, s]\right)\right)_{0}
$$

Now let $s=q / p$ with $p, q \in \boldsymbol{Z}$ coprime. Then we showed in Lemma 3 that the set $\left\{(1),(t),\left(t^{2}\right), \cdots,\left(t^{q-1}\right)\right\}$ is all of the indecomposable free non-isomorphic graded modules. Therefore we have an isomorphism of $S[\mathfrak{P}, s]$-modules;

$$
\boldsymbol{V} \otimes_{R[p, e]} S[\mathfrak{R}, s] \cong(1)^{l_{0}} \oplus(t)^{l_{1}} \oplus\left(t^{2}\right)^{l_{2}} \oplus \cdots \oplus\left(t^{q-1}\right)^{l_{q-1}},
$$

where $l_{i}=\Sigma\left\{m_{j} \mid 0 \leqq j \leqq e-1, j \equiv i(\bmod q)\right\}$. Defining the flag $\overline{\boldsymbol{f}}$ by

$$
\begin{aligned}
\cdots \supset S^{\oplus m} & \supset \mathfrak{P}^{\oplus l_{0}} \oplus S^{\oplus l_{1}} \oplus \cdots \oplus S^{\oplus l_{q-1}} \\
& \supset \mathfrak{P}^{\oplus\left(l_{0}+l_{1}\right)} \oplus S^{\oplus l_{2}} \oplus \cdots \oplus S^{\oplus l_{q-1}} \supset \cdots \\
& \supset \mathfrak{P}^{\oplus\left(l_{0}+l_{1}+l_{2}+\cdots+l_{q-2}\right)} \oplus S^{\oplus l_{q-1}} \supset \mathfrak{P}^{\oplus m} \supset \cdots,
\end{aligned}
$$

we thus show that $B(A, S)=\operatorname{End}_{S}(\overline{\boldsymbol{f}})_{0}$. This implies that $B(A, S)$ is a hereditary $S$-order with ramification index $q$.

REMARK. (i) Generally $A \subset B(A, S) \cap \Sigma$. Thus if $A$ is a maximal order, then $A=B(A, S) \cap \Sigma$.

(ii) Suppose $L / K$ is a finite Galois extension with Galois group $G$. Then $G$ acts on $\Sigma \otimes_{K} L$ naturally, that is, $g(\sigma \otimes l)=\sigma \otimes g(l)(g \in G, \sigma \in \Sigma, l \in L)$. Notice that, in this case, the primes $\left\{\mathfrak{P}_{i 1}, \mathfrak{P}_{i 2}, \cdots, \mathfrak{P}_{i_{i}}\right\}$ lying over $\mathfrak{p}_{i}$ are conjugate by the action of $G$. Hence, $s_{i 1}=s_{i 2}=\cdots=s_{i_{i}}$, and the action of $G$ on $\Sigma \otimes_{K} L$ induces the action on $B(A, S)$.

In this case we can prove:

Proposition 2. Suppose that $L / K$ is a finite Galois extension with Galois group $G$. Then we have $B(A, S)^{G}=A$.

ProOF. We have only to prove the proposition after localizing at $\mathfrak{p} \in X^{1}(R)$. Thus we may assume from Lemma 2 that $(R, \mathfrak{p})$ is a discrete valuation ring, and therefore $\left(S, \mathfrak{B}_{1}, \mathfrak{P}_{2}, \cdots, \mathfrak{P}_{\nu}\right)$ is a semilocal ring.

If $e_{p}=1$, then, since $B(A, S)=A \bigotimes_{R} S$, we have

$$
B(A, S)^{G}=\left(A \otimes_{R} S\right)^{G}=A \otimes_{R} S^{G}=A .
$$

Assume $e_{p}>1$. Then noting that 


$$
\left(\prod_{j=1}^{\nu} \mathfrak{P}_{j}^{[n / 8]}\right) \cap K=\mathfrak{p}^{[[n / s] / r]}=\mathfrak{p}^{[n / e]}
$$

for any $n \in Z$, we show that

$$
A \subset B(A, S)^{G} \subset B(A, S) \cap\left(\Sigma \otimes_{K} K\right)=\sum_{n \in Z} J^{-n} \mathfrak{p}^{[n / e]} \subset A,
$$

hence the proposition.

Also we can show that the extension $B(A, S) / A$ is "Galois" when $S / R$ is nice enough.

THEOREM 2. Suppose the field extension $L / K$ is Galois with Galois group $G$. For any $\mathfrak{p} \in X^{1}(R)$ we assume the following conditions:

(i) If $\mathfrak{p}$ is different from any of $\left\{\mathfrak{p}_{i}\right\}$ (i.e. $e_{\mathfrak{p}}=1$ ), then $R_{\mathfrak{p}} \subset S_{\mathfrak{p}}$ is an unramified extension.

(ii) The characteristic of the field $\kappa(\mathfrak{p})$ is prime with the order $|G|$ of $G$.

(iii) The numbers $s_{i j}$ are integers, i.e. each $r_{i j}$ divides $e_{i}$.

Then there is a natural isomorphism of $R$-algebras:

$$
B(A, S) * G \cong \operatorname{End}_{A}(B(A, S)),
$$

where the left-hand-side denotes the skew group ring of $G$ over $B(A, S)$.

Proof. As before we may assume that $(R, \mathfrak{p})$ is a discrete valuation ring. We write $B=B(A, S)$ for short.

If $e_{p}=1$, then, by the assumption, the natural map $S * G \rightarrow \operatorname{End}_{R}(S)$ is an isomorphism. Thus it follows that

$$
\begin{aligned}
\operatorname{End}_{A}(B) & =\operatorname{End}_{A}\left(A \bigotimes_{R} S\right) \cong A \bigotimes_{R} \operatorname{End}_{R}(S) \\
& \cong A \bigotimes_{R}(S * G)=\left(A \bigotimes_{R} S\right) * G=B * G
\end{aligned}
$$

Suppose $e_{p}>1$. Since $L / K$ is a Galois extension, there is a natural isomorphism $\varphi$ defined as follows :

$$
\varphi: \operatorname{End}_{\Sigma}\left(\Sigma \otimes_{K} L\right) \longrightarrow \Sigma \otimes_{K} \operatorname{End}_{K}(L) \longrightarrow \Sigma \otimes_{K}(L * G)=\left(\Sigma \otimes_{K} L\right) * G .
$$

Note that $\operatorname{End}_{A}(B) \subset \operatorname{End}_{\Sigma}\left(\Sigma \otimes_{K} L\right)$ and $B * G \subset\left(\Sigma \otimes_{K} L\right) * G$. It is clear from the definition of $\varphi$ that $\varphi^{-1}(B * G) \subset \operatorname{End}_{A}(B)$. It is sufficient to show that $\varphi\left(\operatorname{End}_{A}(B)\right)$ $\subset B * G$. To this end, let $G=\left\{g_{1}, g_{2}, \cdots, g_{n}\right\}(n=|G|)$, and for a fixed $f \in$ $\operatorname{End}_{A}(B)$ write

$$
\varphi(f)=\sum_{i=1}^{n} \xi_{i} g_{i} \quad\left(\xi_{i} \in \Sigma \otimes_{K} L\right) .
$$

We want to prove that $\xi_{i} \in B$ for any $i$. For an $R$-free basis $\underline{\lambda}=\left\{\lambda_{1}, \lambda_{2}, \cdots, \lambda_{n}\right\}$ 
of $S$, we set $\Delta(\underline{\lambda})=\operatorname{det}\left(g_{i}\left(\lambda_{j}\right)\right)_{1 \leq i, j \leq n}$. It is well-known that the ideal $\Delta(\underline{\lambda}) S$ is independent of the choice of $\underline{\lambda}$. In fact, $\Delta(\underline{\lambda})^{2} S$ is the discriminant of the extension $S / R$. By [4, Chap. III $\S 6$ Proposition 13] it is also known that

$$
\Delta(\underline{\lambda})^{2} S=\prod_{i=1}^{l} \mathfrak{P}_{i}^{(r-1) n},
$$

where $r$ is the ramification index at $\mathfrak{P}_{i}$ on $\mathfrak{p}$. Let $\mathfrak{Q}=\prod_{i=1}^{l} \mathfrak{P}_{i}$ and $d=\operatorname{dim}_{\kappa(\mathfrak{p})}$ $(S / \mathbb{Q})$. Since $d r=n$, we can take an $R$-free basis $\left\{\lambda_{i} \mid 1 \leqq i \leqq n\right\}$ of $S$ with the property that $\lambda_{i} \in \mathfrak{Q}^{[i / d]-1}-\mathfrak{Q}^{[i / d]}$ for any $i(1 \leqq i \leqq n)$. Then by the definition of $B$ the following are all contained in $B$;

$$
\lambda_{1}, \cdots, \lambda_{d}, J^{-u_{1} \lambda_{d+1}}, \cdots, J^{-u_{1}} \lambda_{2 d}, J^{-u_{2} \lambda_{2 d+1}}, \cdots, J^{-u_{2} \lambda_{3 d}}, \cdots, J^{-u} r-1 \lambda_{n},
$$

where $u_{i}=\max \{u \in \boldsymbol{Z} \mid[u / s] \leqq i\}$. Hence their images under the map $f$ are also in $B$, i.e.

$$
\begin{gathered}
\sum_{i} \xi_{i} g_{i}\left(\lambda_{1}\right), \cdots, \sum_{i} \xi_{i} g_{i}\left(\lambda_{d}\right) \in B \\
J^{-u_{1}} \sum_{i} \xi_{i} g_{i}\left(\lambda_{d+1}\right), \cdots, J^{-u_{1}} \sum_{i} \xi_{i} g_{i}\left(\lambda_{d+2}\right), \cdots, J^{-u_{r-1}} \sum_{i} \xi_{i} g_{i}\left(\lambda_{n}\right) \subset B .
\end{gathered}
$$

Denoting the matrix $\left(g_{i}\left(\lambda_{j}\right)\right)_{1 \leq i, j \leq n}$ by $C$, we thus showed that $\left(\xi_{1}, \xi_{2}, \cdots, \xi_{n}\right) C$ $\in(B)^{\oplus d} \oplus\left(J^{u_{1}} B\right)^{\oplus d} \oplus \cdots\left(J^{u_{r-1}} B\right)^{\oplus d}$. Let $C_{i j}$ be the cofactor of the matrix $C$. Then it follows from the choice of $\left\{\lambda_{i}\right\}$ that

$$
C_{i j} \in \mathbb{Q}^{n(r-1) / 2-[j / d]+1} \text {. }
$$

Therefore we have

$$
\xi_{i} \Delta(\underline{\lambda}) \in \mathbb{Q}^{n(r-1) / 2}\left(B+\mathbb{Q}^{-1} J^{u_{1}} B+\cdots+\mathbb{Q}^{-(r-1)} J^{u_{r-1}} B\right) .
$$

Here, since $s$ is an integer, we note that $\left[-u_{i} / s\right]=-i$ for any $i$. Hence $\mathfrak{Q}^{-i} J^{u_{i}} \subset B$. Combining this with the above we have

$$
\xi_{i} \in \Delta(\underline{\lambda})^{-1} \mathfrak{Q}^{n(r-1) / 2} B=B,
$$

as required.

Finally we give an example.

EXAMPLE. Let $n$ be an integer and $k$ be any field that contains the primitive $n$-th root $\zeta$ of unity. Consider the extension of polynomial rings over $k$ :

$$
R=k\left[x^{n}, y^{n}\right] \subset S=k[x, y] \text {. }
$$

We define an $R$-order $A$ by

$$
A=R\langle u, v\rangle /\left(u^{n}-x^{n}, v^{n}-y^{n}, u v-\zeta v u\right) .
$$

By computing the reduced discriminant of $A$ over $R$ it is easy to check that $A$ 
is ramified only on the primes $\mathfrak{p}_{1}=x^{n} R$ and $\mathfrak{p}_{2}=y^{n} R$, and $n$ is the ramification index of $A$ on each prime. Furthermore $S$ is also ramified on $\mathfrak{p}_{1}$ and $\mathfrak{p}_{2}$ with ramification index $n$. Thus by the definition of blowing-up we have

$$
B(A, S)=\sum_{n_{0}, n_{1}=1}^{n-1}\left\{(u)^{n_{1}}(v)^{n_{2}}(x)^{-n_{1}}(y)^{-n_{2}}\right\}
$$

which is the $k[x, y]$-algebra generated by $\{u / x, v / y\}$. In this case it is also easy to see that $B(A, S)$ is isomorphic to the matrix ring $M_{n}(S)$ by the following mapping :

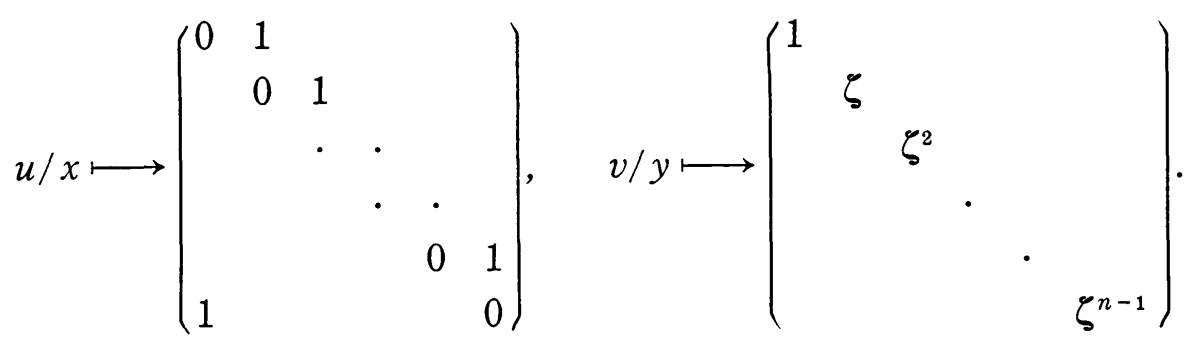

\section{References}

[1] M. Artin, Maximal orders of global dimension and Krull dimension two, Inv. Math. 84 (1986), 195-222; Correction, 90 (1987), p. 217.

[2] L. Le Bruyn, M. Van den Bergh and F. Van Oystaeyen, "Graded Orders", Birkhäuser, Boston, 1988.

[3] I. Reiner, "Maximal Orders", Academic Press, London, 1975.

[4] J.-P. Serre, “Local Fields", Springer Verlag, New York, Heiderberg, Berlin, 1979.

The first author;

Department of Mathematics, Yoshida College

of Kyoto University, Yoshida-Nihonmatsu,

Sakyo-ku, Kyoto 606, JAPAN

The second author;

Department of Mathematics, Nagoya University, Chikusa-ku, Nagoya 464-01, JAPAN 